\title{
UAVs versus Pirates: A Pheromone-based Swarm Monitoring Method
}

\author{
Ruiwen Zhang, Tom Holvoet, Bifeng Song, and Yang Pei
}

\begin{abstract}
As piracy presents great threats to maritime security, unmanned aerial vehicle (UAV) swarm monitoring is becoming an attractive countermeasure. Conventional sweep monitoring method provides balanced coverage but cannot adapt to variable situations, such as the return of UAVs for refueling. This paper proposes a pheromone-based method for UAV swarm anti-piracy monitoring. In this method, the environment is modeled by a pheromone map, and the motion of UAVs is guided by the strength of pheromones. A ship-centered convening mechanism is proposed to increase the chance of finding pirates by taking the interactions between pirates and merchant ships into account. A prediction-reservation mechanism is proposed to improve the deficiencies of the implicit intention propagation mechanism when the ship-centered convening mechanism is used. Simulation experiments are conducted to study the effectiveness of the proposed method. Results show that the proposed method reduces the success rate of pirate attack by $8 \%$ at most compared to the sweep method, and that the prediction-reservation mechanism performs better than the implicit intention propagation mechanism, especially with large number of UAVs.
\end{abstract}

\section{INTRODUCTION}

Contemporary piracy presents great threats to maritime security worldwide. In 2007, more than 320 piracy incidents happened in hot-spot areas including West Africa, East Africa, Asia, and Latin America, causing more than \$2.2 billion in economic losses and $\$ 430$ million in protection expenses [1]. Governments and private agencies have devoted massive efforts in anti-piracy activities, taking countermeasures such as recommended transit corridors, group transit, escorted convoy, coordinated patrol, and on-board security teams [2]. Recently, besides those conventional countermeasures, unmanned aerial vehicles (UAVs) become increasingly attractive anti-piracy instruments. UAVs can be deployed to monitor large areas and act as deterrents by initiating tactical maneuvers to prevent piracy incidents. America, Italy, and Spain have already deployed UAVs in their Somalia escort operations, and private security agencies like ATAC have also started to provide anti-piracy UAV products and services.

As it is required to persistently monitor large areas in anti-piracy missions, a single UAV cannot provide effective monitoring due to the limited operational radius and endurance. Therefore, UAV swarms become better solutions. UAV swarm persistent monitoring has been extensively studied previously. A typical method is the sweep method, in which the area is divided into multiple partitions and each UAV monitors one

Ruiwen Zhang (corresponding author), Bifeng Song, and Yang Pei are with the School of Aeronautics, Northwestern Polytechnical University, Xi'an, 710072 China (phone: 86-29-88494056; e-mail: rwzhangchina@outlook.com, bfsong@nwpu.edu.cn, peiyang_yang@nwpu.eud.cn).

Tom Holvoet is with the Department of Computer Science, Katholieke Universiteit Leuven, Leuven, 3001 Belgium (e-mail: Tom.Holvoet@) cs.kuleuven.be). partition in the sweep motion [3, 4]. The sweep method provides balanced coverage but is less responsive to variable situations such as the return of UAVs for refueling, as area partitioning and sweep path planning are usually designed beforehand.

By contrast, the self-organized method provides adaptive solutions for those variable situations. In the self-organized method, a UAV swarm is regarded as a multi-agent system, in which each UAV dynamically makes its local decisions, and global behaviours emerge as a result of self-organization [5]. The pheromone-based method derived from the foraging behaviour of ant species is a typical type of self-organized method. Ants lay down pheromones as they search for foods. The trails of pheromones then guide other ants to gather to food sources or evade undesirable places. Sauter et al. applied those principles to solve multi-UAV surveillance and tracking problems, proposing the evaporation and propagation mechanisms [6]. Zurad et al. used less kinds of pheromones to coordinate coverage and target detection behaviours [7]. Paradzik et al. proposed to use repulsive planning pheromone to share intended plans [8]. Gao et al. replaced the propagation and evaporation mechanisms with explicitly formulating the variation of pheromones with distance and considered the joining and leaving of UAVs [9].

Although the pheromone-based method has been studied in many applications, the application in anti-piracy scenarios has not been discussed before. With regard to anti-piracy, the monitoring would be more effective if we could take the interactions between pirates and merchant ships into account: as the purpose of pirates is to attack merchant ships, if UAVs focus more on areas surrounding merchant ships, there would be more chances of finding pirates.

In that case, as UAVs are concentrated in smaller areas, the need for propagating the intention of agents to avoid duplicated visits gets more imperative. Previous works used repulsive pheromones to propagate intention, but such implicit mechanism does not include the identity and time information of plans. Claes et al. proposed the delegate multi-agent system method for intention propagation [10]. They used a reservation-based mechanism to share the identity and time information of plans, and achieved more effective coordination in vehicle routing problems.

This paper proposes a pheromone-based method for UAV swarm anti-piracy monitoring. We propose a ship-centered convening mechanism to increase the chances of finding pirates, and a prediction-reservation mechanism to improve intention propagation. The return of UAVs for refueling is also considered. Simulation experiments are conducted to compare the proposed method to the sweep method and the pheromone-based method without the two mechanisms above, demonstrating the effectiveness of the proposed method. 


\section{PROBLEM ForMULATION}

\section{A. Scenarios and Assumptions}

We study a scenario simplified from real situations. The UAV swarm is deployed to monitor a square area without coasts or islands. Assume the station is located nearby and outside the area. UAVs are small fixed wing propeller aircraft equipped with camera sensors. UAVs maintain level flight at the constant velocity and altitude. The turning radius is negligible. The camera has a fixed field of view (FOV), and points downwards in the symmetry plane of aircraft. UAVs can communicate with the station and each other all over the area with negligible latency. UAVs depart from the station and return for refueling when the remaining endurance is barely sufficient to return. Merchant ships cross the area at constant velocities in straight lines. Pirates know the routes of merchant ships beforehand. After entering the area, a pirate intercepts a random merchant ship at a constant velocity in a straight line.

We assume that when a UAV detects a pirate, it deters the pirate by circle around it. From that signal the pirate knows it has been noticed, and leaves the area immediately. The modeling of the circling flight is ignored. If a pirate can reach a merchant ship without being noticed, it will be regarded as a successful attack. The velocities of merchant ships and pirates follow the uniform distribution in given ranges. The directions of merchant ships follow the uniform distribution in the range of $\left[0,360^{\circ}\right)$. The entering positions of merchant ships and pirates are uniformly distributed on the edges of the area, and the arrival rates follow the Poisson distribution.

\section{B. Metrics}

We use the success rate of attack to evaluate the effectiveness of monitoring, expressed as

$$
r_{\text {attack }}=\frac{n_{\text {attack }}}{n_{\text {pirate }}}
$$

where $n_{\text {attack }}$ is the number of successful attacks, and $n_{\text {pirate }}$ is the total number of pirates entering the area. In addition, the revisit interval, which is the time difference between two adjacent visits of a position, is also an important metric to consider, as shorter revisit intervals usually mean higher chances of finding pirates. To quantify the revisit interval, the area is discretized into a grid map. When the center of a cell is in the FOV of a UAV and the projection center of the camera is in the cell at the same time, the cell will be regarded as visited. The time average of maximum revisit intervals is regarded as the evaluation metric, expressed as

$$
\begin{gathered}
\mu_{\text {tam }}=\frac{1}{T_{m}} \int_{0}^{T_{m}} \mu_{\max }(t) d t, \\
\mu_{\max }(t)=\max _{1 \leq c \leq n_{c}}\left\{\mu_{c}(t)\right\},
\end{gathered}
$$

where $n_{c}$ is the total number of cells, $c$ is the index of cells, $\mu_{c}(t)$ is the revisit interval of $c$ th cell at time $t, \mu_{\max }(t)$ is the maximum revisit interval of the area at time $t$, and $T_{m}$ is the duration of monitoring. The time average of maximum revisit intervals will be abbreviated as the maximum revisit interval below.

The demonstration of the scenario and area discretization is shown Fig. 1.

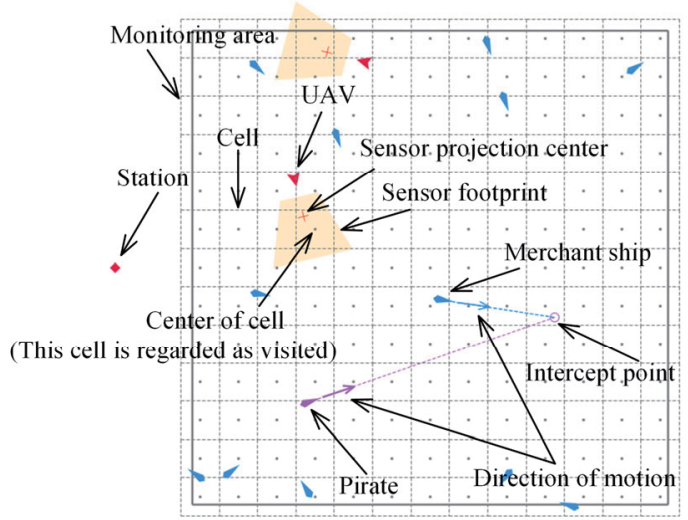

Figure 1. Demonstration of the problem formulation

\section{PROPOSED APPROACH}

\section{A. Models of Agent Motion and Sensing}

The motion of agents is modeled by 3-degree-of-freedom kinetic equations in continuous time and 3-dimension space, described in [11]. The geometry of UAV agent sensing is shown in Fig. 2. $h_{f}$ is the flight altitude, $\vartheta_{h}$ and $\vartheta_{v}$ are the horizontal and vertical FOV respectively, $\lambda$ is the look-down angle, $x_{b}$ is the longitudinal axis of aircraft, $x_{s}$ is the normal axis of the focal plane, $P_{s}$ is the projection center, and $w_{\mathrm{s}}$ is regarded as the sweep width.

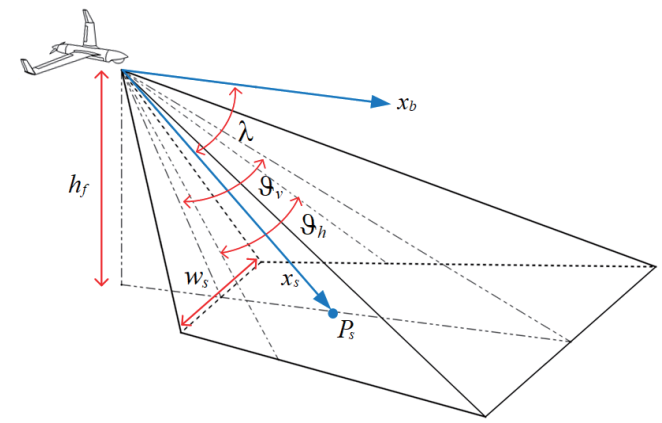

Figure 2. Geometry of the agent sensing

The probability of detection depends on the parameters of focal plan arrays (FPAs) and the distance between the target and the sensor, described in [12]. The $m / n$ logic sliding windows criteria, described in [13], is used to determine whether a target is detected.

\section{B. Pheromone-based Monitoring Method}

In the pheromone-based monitoring method, the environment is discretized into a grid map. Each cell has a certain amount of pheromones, and the motion of agents is guided by the strength of pheromones. Pheromones are usually classified into two characters: attractive pheromones attract agents towards the cells, and repulsive pheromones repulse agents away from the cells. 
The area is discretized in the same way as described in section II B. Assume the map is shared among all agents. The map could be managed by certain agents, either the station or UAVs, and transmitted to others when required. The map updates in the period of $T_{u}$, and the resulting discretized time step is denoted as $\tau$. Two kinds of pheromones are used: visited pheromones and convening pheromones. Visited pheromone are repulsive. Agents assign a base value of pheromones $\varphi_{0}$ when they visit a cell. Then the value of visited pheromones of a cell at the time step $\tau$ is given by

$$
\varphi_{v}(\tau)=\varphi_{0} \cdot\left(1-e_{v}\right)^{\tau-\tau_{k}}, \tau \geq \tau_{k},
$$

where $e_{v}$ is the attenuation coefficient of visited pheromones, and $\tau_{k}$ is the time step when the cell is most recently visited.

Convening pheromones are attractive and mark cells that need to visit. When a cell is unvisited for a given time steps $\tau_{c}$, the cell becomes a convening cell, and $\tau_{c}$ is called the convening interval. The convening cell creates a field of convening pheromones until it is visited again. The value of convening pheromones of a cell in the field is given by

$$
\varphi_{c}(\delta)=\frac{c_{c 1}}{c_{c 2}+\delta}+c_{c 3},
$$

where $\delta$ is the distance to the convening cell measured in the number of cells. The convening coefficients $c_{c 1}, c_{c 2}$, and $c_{c 3}$ are solved by specifying the values of $\varphi_{c}$ at three specific distances, given by

$$
\left\{\begin{array}{l}
\varphi_{c}(0)=\varphi_{0} \\
\varphi_{c}(1)=\left(1-e_{c}\right) \cdot \varphi_{0}, \\
\varphi_{c}\left(\sqrt{2} \delta_{m}\right)=0
\end{array},\right.
$$

where $e_{c}$ is the attenuation coefficient of convening pheromones, and $\delta_{m}$ is the maximum convening distance. The field remains constant with time and can be superposed. The pheromone sum is given by

$$
\varphi_{s}=\varphi_{c}-\varphi_{v} .
$$

The lower limit for each kind of pheromone is 0 , and the value below a small number $\varepsilon_{\varphi}$ will be regarded as zero. An upper limit $\varphi_{m}$ is set to prevent excessively large or small values.

The agents plan paths with a rolling horizon. A cell planned to visit is called a plan step. When the first plan step is visited, a new plan step will be added, maintaining a fixed number of plan steps $n_{p}$ in store. When calculating new plan steps, the last plan step at the moment is called the leading cell.

Paths are planned by the greedy algorithm. The agent chooses the cell with the highest value of pheromone sums in the Moore neighbors of the leading cell as the next plan step. When the values are the same in two cells, the cell requiring a smaller turning angle will be chosen. Then the center of the cell will be used to calculate the flight path. The demonstration of the pheromone-based monitoring method is shown in Fig. 3.

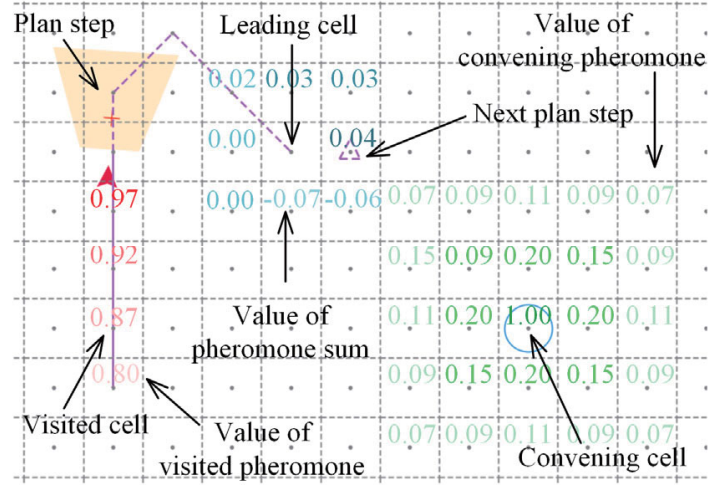

Figure 3. Demonstration of the pheromone-based monitoring method

\section{Ship-Centered Convening Mechanism}

In the general convening mechanism described in III B, any cells can become convening cells when the revisit intervals measured in time steps exceed $\tau_{c}$. We propose a ship-centered convening mechanism to attract agents to areas around merchant ships. In the ship-centered convening mechanism, only the cells whose distances to a merchant ship are in a given range $\left[d_{\text {emin }}, d_{\text {emax }}\right]$ can become convening cells when the revisit intervals measured in time steps exceed $\tau_{c} . d_{\text {emin }}$ and $d_{\text {emax }}$ are called the minimum and maximum escort distance respectively. The demonstration of the ship-centered convening mechanism is shown in Fig. 4.

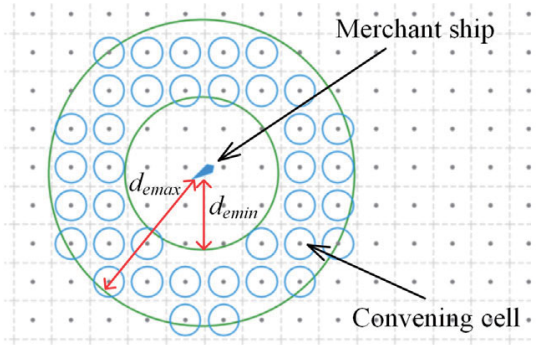

Figure 4. Demonstration of the ship-centered convening mechanism

\section{Prediction-Reservation Intention Propagation Mechanism}

In the implicit intention propagation mechanism, agents assign a certain amount of another kind of repulsive pheromone called planning pheromone in plan steps to reduce duplicated visits. The assignment is periodical, and the value decreases with time, so that planning pheromones in abandon plan steps will vanish after a while. In our method the initial value assigned is $\varphi_{0}$, and the decrease with time is given by

$$
\varphi_{p}(\tau)=\varphi_{p}(\tau-1)-\Delta \varphi_{p},
$$

where $\varphi_{p}(\tau)$ is the value of planning pheromones at time step $\tau$, and $\Delta \varphi_{p}$ is the decrement. Planning is based on the current pheromone values of cells.

We propose a prediction-reservation mechanism to achieve explicit intention propagation. Each cell records a piece of reservation information $\left\langle A_{r}, \tau_{r}\right\rangle$, where $A_{r}$ is the reservation maker, and $\tau_{r}$ is the time step when $A_{r}$ is predicted to be visited, named as the reservation time. When planning, agents 
first predict the time steps to visit candidate cells and the value of pheromones in those cells at that time step using equations (4) $\sim(7)$. Then the next plan step is chosen based on those predicted values, and a reservation is made accordingly.

If there is a reservation before the predicted visiting time step, the reservation time will be regarded as the visited time when predicting the values of pheromones. In planning, the predicted value of pheromones and the reservation maker, if any, will be recorded. When adding new plan steps, agents first re-predict the value of pheromones and check the reservation maker of existing plan steps. If the variation of pheromone sum exceeds a given threshold $\sigma_{\varphi}$, called the replanning threshold, or the reservation maker changes, the subsequent plan steps will be cancelled and new plan steps will be added.

Only the reservations with the earliest reservation time are recorded, and a reservation can only be made if there is no reservation or the reservation time is earlier than the existing one. If a plan step is cancelled, agents managing the map will be notified, and the reservation will be cancelled accordingly.

\section{SimUlATION EXPERIMENTS}

\section{A. Simulation Settings}

The area is $100 \mathrm{~km} \times 100 \mathrm{~km}$. The station is located at $5 \mathrm{~km}$ perpendicularly away from the center of one edge. The duration of monitoring is 7 days. The endurance of UAVs $E_{a}$ is 6 hours, and UAVs return for refueling when the remaining endurance is barely sufficient to return. The refueling takes 1 hour, then the UAV is ready to be dispatched again. UAVs are dispatched in a fixed interval $\Delta t_{a d}$, and the number of UAVs in store is sufficient. If it is required to keep $n_{a}$ UAVs in the air at any time, $\Delta t_{a d}$ will be given by

$$
\Delta t_{a d}=E_{a} / n_{a} .
$$

The velocity of UAVs $v_{a}$ is $30 \mathrm{~m} / \mathrm{s}$, and the flight altitude is $2500 \mathrm{~m}$. The velocity ranges of merchant ships and pirates are $[5,12]$ and $[12,18]$ in $\mathrm{m} / \mathrm{s}$ respectively. The average arrival intervals of merchant ships and pirates are 5 minutes and 12 hours respectively. The parameters of the camera are shown in Table I.

TABLE I. PARAMETERS OF CAMERA

\begin{tabular}{|l|c|}
\hline \multicolumn{1}{|c|}{ Parameters } & Values \\
\hline FPA size $(\mathrm{mm} \times \mathrm{mm})$ & $4.8 \times 3.6$ \\
\hline FPA resolution $($ pixel $\times$ pixel) & $1296 \times 736$ \\
\hline FOV, horizontal $\times$ vertical $\left({ }^{\circ} \times{ }^{\circ}\right)$ & $51.3 \times 39.6$ \\
\hline Focal length $(\mathrm{mm})$ & 5 \\
\hline Look-down angle $\left({ }^{\circ}\right)$ & 45 \\
\hline
\end{tabular}

The width of cells $w_{c}$ equals the sweep width $w_{s}$. The update period of the map is given by

$$
T_{u}=0.5 \cdot w_{c} / v_{a} .
$$

The convening interval in the general convening mechanism $\tau_{c, g}$ is given by

$$
\tau_{c, g}=\left\lfloor 1.5 \cdot n_{c} \cdot w_{c} /\left(n_{a} \cdot v_{a} \cdot T_{u}\right)\right\rfloor,
$$

where $\lfloor\cdot\rfloor$ is the floor function. The convening interval in the ship-centered mechanism is given by

$$
\tau_{c, s}=\left\lfloor 1.5 \cdot n_{c e} \cdot w_{g} /\left(v_{a} \cdot T_{u}\right)\right\rfloor,
$$

where $n_{c e}$ is the number of cells in the escort range. The base value of pheromones $\varphi_{0}$ is 1 , the upper limit $\varphi_{m}$ is 10 , and the small number regarded as zero $\varepsilon_{\varphi}$ is $1 \times 10^{-4}$. The attenuation coefficient of visited pheromones $e_{v}$ is given by

$$
e_{v}=1-\left(\varepsilon_{\varphi} / \varphi_{0}\right)^{1 / \tau_{c}},
$$

where $\tau_{c}$ is $\tau_{c, g}$ or $\tau_{c, s}$ in respective convening mechanisms. The attenuation coefficient of convening pheromones $e_{c}$ is 0.2 , and the maximum convening distance is given by

$$
\delta_{m}=\left\lfloor\sqrt{n_{c} / n_{a}}\right\rfloor .
$$

The decrement of planning pheromones $\Delta \varphi_{p}$ is $\varphi_{0} / 3$. The escort range is $[5,10]$ in $\mathrm{km}$.

The simulation is run on the Anylogic simulation platform. The demonstration of simulation after 3 days is shown in Fig. 5. The trails of 8 UAVs are shown in Fig. 5 (a). The distributions of revisit intervals with $20 \mathrm{UAVs}$ in the air in the air are shown in Fig. 5 (b) and (c). General convening brings about more balanced coverage, while ship-centered convening makes the cells around merchant ships be visited more frequently, leaving the cells with less merchant ships unvisited for a long time.

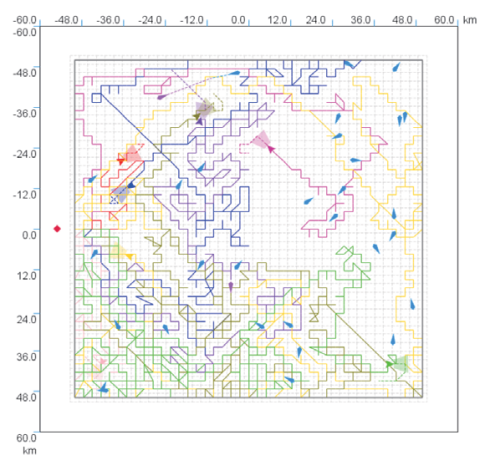

(a) Trails of UAVs

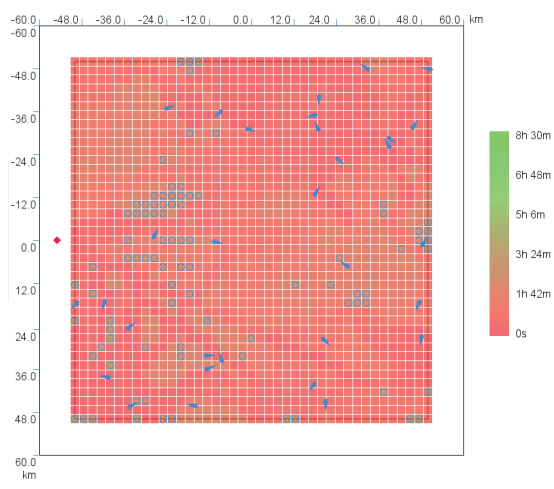

(b) Distribution of revisit intervals (general convening) 


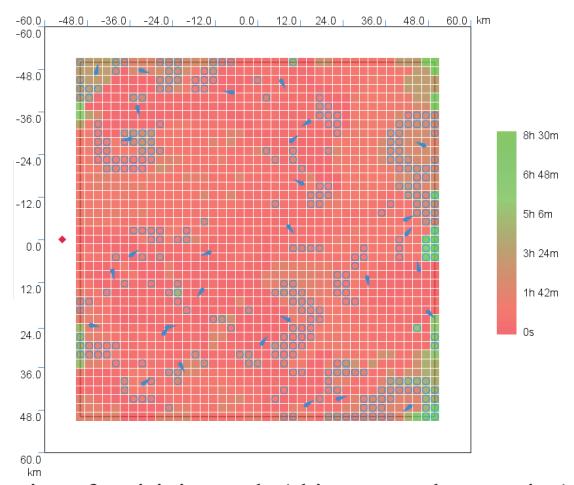

(c) Distribution of revisit intervals (ship-centered convening)

Figure 5. Demonstration of simulation

\section{B. Experiments and Results}

In pheromone-based methods, the choice of planning parameters is an important issue. A lower replanning threshold makes agents more responsive to the variation of situations, while a higher threshold avoids the instability caused by frequently changing plans. More plan steps reduce conflicts by propagating more intentions, while less plan steps make decisions precise as longer horizons will induce more prediction errors.

We study the following 4 cases:

- using planning pheromones, denoted as "PP";

- using the prediction-reservation mechanism with $\sigma_{\varphi}=0.5$, denoted as "PR 0.5 ";

- using the prediction-reservation mechanism with $\sigma_{\varphi}=1$, denoted as "PR 1";

- using the prediction-reservation mechanism with infinite large $\sigma_{\varphi}$, denoted as "PR $\infty$ ".

The infinite large threshold means no response to the variation of predicted pheromone values. The number of plan steps is increased from 1 to 20 . The number of UAVs in the air is 10 , and the general convening mechanism is used. For each sample the simulation is run for 200 replications.

Results are shown in Fig. 6. For all the cases, with increasing number of plan steps, the maximum revisit interval $\mu_{\text {tam }}$ declines at first and then rises. The rise is especially sharp in the case using planning pheromones, as planning is based on current pheromone values, whose deviations from the future values when the cells will be visited enlarge with the increasing number of steps, leading to less effective paths. However, in the cases using the prediction-reservation mechanism, planning is based on the pheromone values at the time steps that the cells are predicted to be visited, so the deviations are much smaller. Therefore, a medium number of steps around 10 is suitable. For the prediction-reservation mechanism, $\mu_{\text {tam }}$ is generally shorter when the replanning threshold is higher. Therefore, a higher threshold is preferred, indicating that stability outweighs responsiveness. The variation of success rates of attack $r_{\text {attack }}$ does not have clear indications.

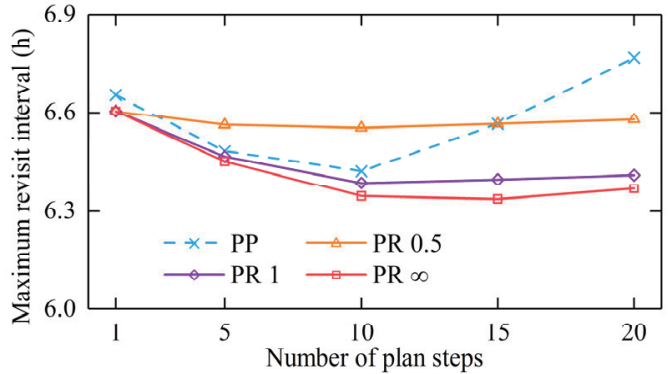

(a) Maximum revisit interval

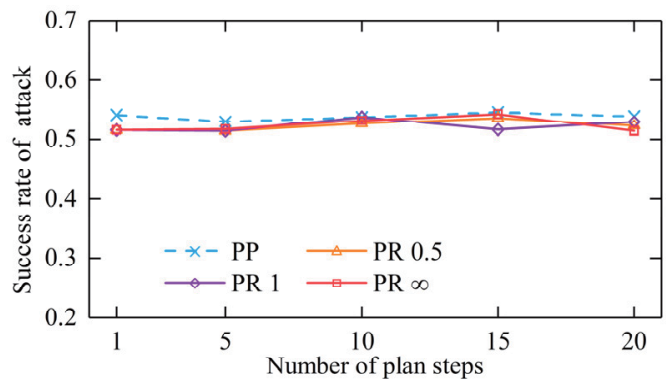

(b) Success rate of attack

Figure 6. Effects of planning parameters

The difference of effectiveness among monitoring methods is affected by the number of UAVs, because increasing the number leads to rising conflicts of intentions, and the ability of reducing conflicts is different among intention propagation mechanisms. Therefore, we compare the proposed method to the sweep method and pheromone-based methods without proposed mechanisms while varying the number of UAVs. Details of the sweep method are described in [3, 4]. In the sweep method, when a UAV returns, the respective area partition of it is left unmonitored until another UAV is dispatched there, while in pheromone-based methods, UAVs autonomously adapt to the situation by the variation of pheromones. We study the following 5 cases:

- the sweep method, denoted as "SW";

- the pheromone-based method using planning pheromones and the general convening mechanism, denoted as "PPG";

- the pheromone-based method using the predictionreservation mechanism and the general convening mechanism, denoted as "PRG";

- the pheromone-based method using planning pheromones and the ship-centered convening mechanism, denoted as "PPS";

- the pheromone-based method using the predictionreservation mechanism and the general convening mechanism, denoted as "PRS".

The number of UAVs is increased from 8 to 20, considering the assurance of coverage and the viable cost and maintainability. The number of plan steps in pheromone-based methods is 10, and the replanning threshold in prediction-reservation mechanisms is 1 . For each sample the simulation is run for 200 replications. The maximum revisit interval does not have meaningful indications when using the 
ship-centered mechanism as the distribution is highly unbalanced, so it is not analyzed in the case "PPS" and "PRS".

Results are shown in Fig. 7. Within the feasible range of the number of UAVs in the air $n_{a}$, increasing the number reduces success rates of attack $r_{\text {attack }}$ and maximum revisit intervals $\mu_{\text {tam }}$. For maximum revisit intervals, pheromone-based methods reduce $\mu_{\text {tam }}$ by 3 hours on average compared to the sweep method. Using the prediction-reservation mechanism brings out shorter intervals than using planning pheromones, and the difference enlarges with increasing $n_{a}$. For the success rate of attack, the combination of the two proposed mechanisms reduces $r_{\text {attack }}$ by $8 \%$ at most compared to the sweep method. When the ship-centred convening mechanism is used, using the prediction-reservation mechanism generally brings out lower $r_{\text {attack }}$ than using planning pheromones. Especially when the $n_{a}$ reaches 20, using planning pheromones will barely decrease $r_{\text {attack }}$ while using the prediction- reservation mechanism will continue to decrease $r_{\text {attack }}$. In that case UAVs are more concentrated, and that makes the advantages of prediction-reservation mechanism more prominent.

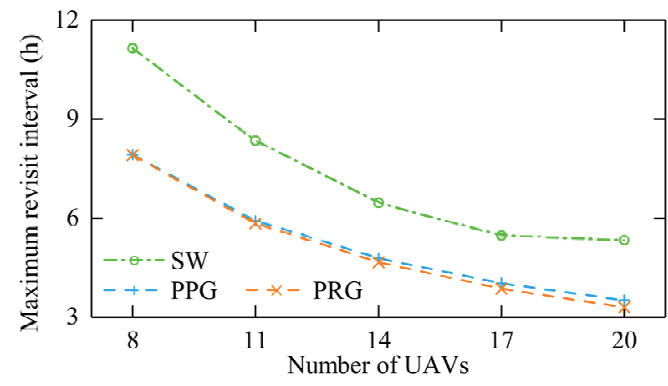

(a) Maximum revisit interval

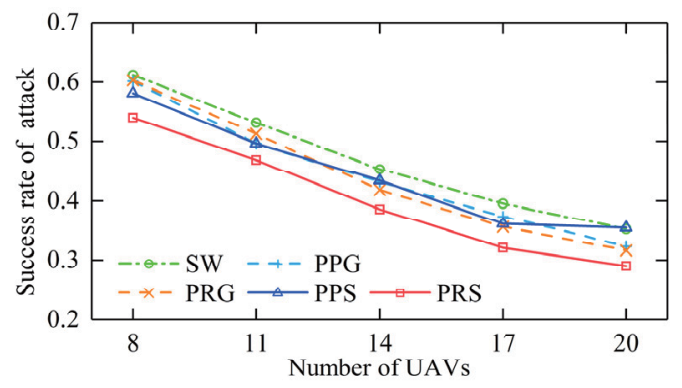

(b) Succes rate of attack

Figure 7. Effectiveness of monitoring methods

\section{CONCLUSION}

This paper studies the issue of using UAV swarms in maritime monitoring to detect and deter pirates. We propose a pheromone-based swarm monitoring method, which takes the interactions between pirates and merchant ships into account by a ship-centered convening mechanism and improves the deficiencies of intention propagation in previous works by a prediction-reservation mechanism. The results of simulation experiments show that the combination of the two proposed mechanisms improves the effectiveness of monitoring, and that the prediction-reservation mechanism prevents the performance degradation of the implicit intention propagation mechanism when the ship-centered convening mechanism is used with large number of UAVs.

As the proposed method is adaptive and extendable, we expect it to demonstrate more advantages in situations with more variability. For example, when the distributions of pirates and merchant ships are not uniform but concentrated in certain areas, or the possibility of being attacked is different among merchant ships, the proposed method can adapt to those variations by changing convening cells and convening intervals. Besides, we only study the feasible range of the number of UAVs, within which the effectiveness is persistently improved by increasing the number. We speculate that the effectiveness will not be improved anymore when the number exceeds a certain value beyond the range above due to excessive conflicts of intentions, and there may be an optimal number. Furthermore, in this paper we only study a simplified scenario that is almost ideal. More analyses can be considered in future works, such as the effect of changing velocity of merchant ships or pirates. Those issues will be the directions to conduct further research.

\section{REFERENCES}

[1] L. Joubert, The State of Maritime Piracy 2017: Assessing the Economic and Human Cost. Broomfield, USA: One Earth Future Foundation, 2018.

[2] O. Vaněk, M. Jakob, O, Hrstka, and M. Pěchouček, "Agent-based model of maritime traffic in piracy-affected waters," Transportation Research Part C: Emerging Technologies, vol. 36, pp. 157-176, Nov 2013.

[3] J. J. Acevedo, B. C. Arrue, I. Maza, and A. Ollero, "Distributed cooperation of multiple UAVs for area monitoring missions," in Motion and Operation Planning of Robotic Systems: Background and Practical Approaches, G. Carbone, and F. Gomez-Bravo, Ed. Cham, Switzerland: Springer, 2015, pp. 471-494.

[4] Y. Li, H. Chen, J. E. Meng, and X. Wang, "Coverage path planning for UAVs based on enhanced exact cellular decomposition method," Mechatronics, vol. 21, no. 5, pp. 876-885, Aug 2011.

[5] M. Senanayake, I. Senthooran, J. C. Barca, H. Chung, J. Kamruzzaman, and M. Murshed, "Search and Tracking Algorithms for Swarms of Robots: A Survey," Robotics and Autonomous Systems, vol. 15, part. B, pp. 422-434, Jan 2016.

[6] J. A. Sauter, R. Matthews, H. Van Dyke Parunak, and S. A. Brueckner, "Performance of Digital Pheromones for Swarming Vehicle Control," Proc. 4th Int. Joint. Conf. Autonomous Agents and Multiagent Aystems, New York, 2005, pp. 903-910

[7] M. R. Brust, M. Zurad, L. Hentges, L. Gomes, G. Danoy and P. Bouvry, "Target tracking optimization of UAV swarms based on dual-pheromone clustering," Proc. 2017 3rd IEEE Int. Conf. Cybernetics, Piscataway, U.S., 2017, pp. 1-8.

[8] M. Paradzik, and G. Ince, "Multi-agent search strategy based on digital pheromones for UAVs," 24th Signal Processing and Communication Application Conf., Piscataway, U.S., 2016, pp. 233-236.

[9] C. Gao, Z. Zhen, and H. Gong, "A self-organized search and attack algorithm for multiple unmanned aerial vehicles," Aerospace Science and Technology, vol. 54, pp. 229-240, July 2016.

[10] R. Claes, T. Holvoet and D. Weyns, "A Decentralized approach for anticipatory vehicle routing using delegate multiagent systems," in IEEE Trans. Intelligent Transportation Systems, vol. 12, no. 2, pp. 364-373, June 2011

[11] R. Zhang, B. Song, Y. Pei, W. Tang, and M. Wang, "Agent-based analysis of multi-UAV area monitoring mission effectiveness," Proc. AIAA Modeling and Simulation Technologies Conf., Reston, USA, 2017, pp. $1-10$

[12] J. Gundlach, Designing Unmanned Aircraft Systems: A Comprehensive Approach, Reston, USA: AIAA, 2012, pp. 552-562.

[13] Y. He, J. Xiu, and X. Gua, Radar Data Processing with Applications, Singapore: John Wiley \& Sons, 2016, pp. 118. 